\title{
Special issue on security, usability and sustainability of smart cities
}

\author{
Hissam Tawfik ${ }^{1} \cdot$ Jia Hu ${ }^{2} \cdot$ Kevin Kam Fung Yuen ${ }^{3} \cdot$ Mohammed M. Alani ${ }^{4} \cdot$ Nektarios Georgalas $^{5}$
}

Published online: 14 February 2020

(c) Springer Nature Switzerland AG 2020

Society and humans have gained real benefits from multidisciplinary research on intelligent environments. By combining real-time information and historical data, intelligent environments make beneficial decisions for their users, which can be useful for smart cities and sustainable development. Specifically, a smart city can utilise intelligent environments to make more efficient use of traditional systems, networks and services, to deliver citizen-centred services. These intelligent systems pose new performance- and reliability-related challenges in terms of usability, security, and dependency, among other challenges. This special issue addresses some of the issues surrounding the developments, modelling, tools, design methodologies, case studies and trend-setting ideas in the areas of security, performance, usability and sustainability of intelligent environments for smart cities.

This special issue features four selected high-quality papers after a rigorous peer-review process. The first paper entitled "End to end light weight mutual authentication scheme in IoT based healthcare environment" proposed an end-to-end mutual authentication scheme to ensure the security and privacy of the patient balancing security and performance. The security analysis shows that the proposed scheme is more efficient compared with other related schemes.

In the paper entitled "Model-Based Testing of Autonomous Robots Using TestIt", the authors present a tool TestIt, for automated model-based testing of autonomous multi-robot systems to verify long-term autonomy in various applications including those of smart city environments

Hissam Tawfik

h.tawfik@leedsbeckett.ac.uk

1 School of Built Environment, Engineering and Computing, Leeds Beckett University, Leeds, UK

2 Department of Computer Science, University of Exeter, Exeter, UK

3 Singapore University of Social Sciences, Singapore, Singapore

4 Senior Member of the ACM, Abu Dhabi, UAE

5 Applied Research Department, British Telecom (BT), Martlesham, UK and smart buildings. The usability of TestIt is demonstrated using a smart building robotic security system case study.

The next paper entitled "Repeatable Determinism using Non-Random Weight Initialisations in Smart City Applications of Deep Learning" uses a variety of non-random weight initialisation schemes in neural networks to make the network more deterministic in learning sessions. This is a particularly desirable property in safety critical systems where deep learning is applied to smart city applications and where public liability is a concern.

The final paper entitled "A novel application of deep learning with image cropping: a smart cities use case for flood monitoring" develops a hybrid approach to utilise deep learning-based image classification with an IoT-enabled camera to monitor gullies and drains. This paper outlines the lessons from experiments that have a wider impact on many similar use cases involving IoT-based cameras as part of smart city event monitoring platforms.

Acknowledgements The guest editors are grateful to the reviewers for their efforts in reviewing the manuscripts. We also thank the Editorsin-Chief, Prof. Juan Carlos Augusto and Prof. Antonio Coronato, for their support and guidance during the entire process.

Publisher's Note Springer Nature remains neutral with regard to jurisdictional claims in published maps and institutional affiliations. 